\title{
KNOWLEDGE ABOUT MENARCHE AND MENSTRUATION, AMONG TRIBAL FEMALES OF KARGIL
}

\author{
SARIKA MANHAS ${ }^{1}$, SABIYA ASMAT ${ }^{2}$ \& TASHI DOLKER ${ }^{3}$ \\ ${ }^{I}$ Assistant professor. P. G., Department of Home Science, University of Jammu, Jammu and Kashmir, India \\ ${ }^{2}$ Subject Matter Specialist, SK University of Agriculture Science \& Technology of Kashmir, Krishi Vigyan Kendra,
} Kargil, Jammu and Kashmir, India

${ }^{3}$ Research Scholar, Department of Home Science, University of Kashmir, Jammu and Kashmir, India

The present study was carried out, to assess the knowledge of tribal females of Kargil, about menstruation. The sample for the study consisted of 320 adolescent's girls, 100 mothers and 100 grandmothers, belonging to Balti and Purig tribes. The random sampling technique was used, to select the sample. A self devised interview schedule was used, to gather the required information. The results reveal that, there is a substantial lacuna in the knowledge about menstruation, among the females. Widespread misconceptions and unawareness about the essential concepts of reproductive physiology and menstruation, among the tribal females was noted. It was found that, all mothers and the majority of grandmothers and adolescent girls, held incorrect perception, regarding the causes of menstruation. For them, menstruation occurs in females, in order to get rid of spoiled or dirty blood, from the body and it helps to detoxify our body. Many of these females were still not sure, about the source of menstrual blood. These girls were not able to differentiate between urethra and vaginal opening. The results overall highlight that, even today females of Kargil, tend to have insufficient and incomplete information, related to menarche and menstruation.

KEYWORDS: Tribal Adolescent Girls, Knowledge, Menstruation \& Menarche
\end{abstract}

Received: Aug 06, 2017; Accepted: Sep 15, 2017; Published: Oct 26, 2017; Paper Id: IJASRDEC201713

\section{INTRODUCTION}

Menstruation is a phenomenon, unique to the females; the onset of menstruation is one of the most important land-mark in the process of growth and maturation (Kamaljit, 2012). Menstruation, the periodic vaginal bleeding, that occurs with the shedding of the uterine mucosa is one of the signs of puberty, and occurs one or two years, following the appearance of secondary sexual characteristics (Shokry, 2011). Menarche is the first menstrual period, or first menstrual bleeding. From both social and medical perspective, it is often considered the central event of the female puberty and it signals the possibility of fertility (Rierdan \& Koff, 1985).

Adolescent girls constitute a vulnerable group, particularly in India, where female child is often neglected. Female young girls' reproductive health is poorly understood and ill served in India. In this age group, girls are growing and have inadequate awareness of reproductive health mechanism. They are also not aware about how to maintain hygienic practices, during menstruation (Kamaljit, 2012). Unawareness has led to many kinds of practices, especially among the school girls, some of which are very much damaging. Adolescent girls often lack knowledge regarding menstruation, which can be due to socio-cultural barriers, in which they grow up (Subhash, 2011). Moreover, reproductive tract infections (RTIs) are more prevalent in this age group. Research has shown that, 
ignorance and lack of preparation around puberty and menstruation perpetuates myths, and leaves the girls vulnerable to feelings of shame, and low self-esteem (Rodriguez, 2012). Incomplete knowledge about menstruation, along with lack of menstrual hygiene, leads to many reproductive infections. The manner in which a girl learns about menstruation and its associated changes, may have an impact process; it is linked with several misconceptions and inadequate practices, which sometimes result in adverse health outcomes (Kamaljit, 2012). When menstrual information is inaccurate and inadequate, it affects women's ability to understand their own reality. Adolescent girls need to acquire the right information, about pubertal reproductive issues at the right age, in order to develop responsible behaviour towards reproductive process, especially in tribal, rural areas like Kargil, where education and source of information is very poor. It is in this context that, the present study assumes importance.

\section{RESEARCH METHODOLOGY}

\section{Sample and Sample Size}

The sample for the study was divided into 3 groups:

\section{Group I}

Comprised of 320 unmarried adolescent girls, in the age group of 14-18 years, selected equally from Balti tribe and Purig tribe, of Kargil District.

\section{Group II}

Consisted of 100 mothers (50 from Purig tribe and 50 from Balti tribe), who had at least one adolescent daughter, who were again selected randomly and equally, from the two tribes.

\section{Group III}

Consisted of 100 grandmothers (50 from Purig tribe and 50 from Balti tribe), who had at least one adolescent granddaughter experiencing menstruation, drawn randomly from Kargil block and Sankoo block of Kargil District. A self structured interview schedule was used, to assess the knowledge of sample, females regarding menarche and menstruation.

\section{RESULTS AND DISCUSSIONS}

\section{The Results of the Study are discussed and presented as follows}

\section{Causes of Occurrence of Menstruation}

Table 1: Causes of Occurrence of Menstruation

\begin{tabular}{|c|c|c|c|c|c|c|c|c|c|c|c|c|c|c|c|}
\hline \multirow[b]{2}{*}{ Causes } & \multicolumn{2}{|c|}{ Purig Girls } & \multicolumn{2}{|c|}{ Balti Girls } & \multirow{2}{*}{\begin{tabular}{|c}
$\begin{array}{c}\text { Early } \\
\text { Ado. }\end{array}$ \\
$14-16$ \\
Years \\
$\mathrm{N}=164$
\end{tabular}} & \multirow{2}{*}{$\begin{array}{c}\begin{array}{c}\text { Late } \\
\text { Ado }\end{array} \\
\text { 16-18 } \\
\text { Years } \\
\mathrm{N}=156\end{array}$} & \multicolumn{2}{|c|}{ Girls } & \multicolumn{2}{|c|}{ Mothers } & \multicolumn{2}{|c|}{ Grandmothers } & \multicolumn{3}{|c|}{ Females } \\
\hline & $\begin{array}{c}14-16 \\
N=77 \\
(\%)\end{array}$ & $\begin{array}{l}16-18 \\
N=83 \\
(\%)\end{array}$ & $\begin{array}{l}14-16 \\
N=87 \\
(\%)\end{array}$ & $\begin{array}{l}16-18 \\
N=73 \\
(\%)\end{array}$ & & & $\begin{array}{l}\text { Purig } \\
\mathrm{N}=160\end{array}$ & $\begin{array}{r}\text { Balti| } \\
\mathrm{N}=160\end{array}$ & $\begin{array}{l}\text { Purig } \\
\mathrm{N}=50\end{array}$ & $\begin{array}{l}\text { Balti } \\
\mathrm{N}=50\end{array}$ & $\begin{array}{c}\text { Puri } \\
\mathrm{g} \\
\mathrm{N}=5 \\
0\end{array}$ & $\begin{array}{l}\text { Balti } \\
\mathrm{N}=\mathbf{5 0}\end{array}$ & $\begin{array}{c}\text { Girls } \\
\mathrm{N}=320\end{array}$ & $\begin{array}{c}\text { Mothers } \\
\mathrm{N}=100\end{array}$ & $\begin{array}{c}\text { Grand } \\
\text { mothers } \\
\mathrm{N}=100\end{array}$ \\
\hline Aging & $\begin{array}{c}36 \\
(46.7)\end{array}$ & $\begin{array}{c}44 \\
(53.0)\end{array}$ & $\begin{array}{c}\mathbf{5 0} \\
(\mathbf{5 7 . 4})\end{array}$ & $\begin{array}{c}21 \\
(28.7)\end{array}$ & $\begin{array}{c}86 \\
(52.4)\end{array}$ & $\begin{array}{c}65 \\
(41.6)\end{array}$ & $\begin{array}{l}80 \\
(50)\end{array}$ & $\begin{array}{c}71 \\
(44.3)\end{array}$ & $\begin{array}{c}\mathbf{5 0} \\
(100)\end{array}$ & $\begin{array}{c}50 \\
(100)\end{array}$ & $\begin{array}{c}35 \\
(70)\end{array}$ & $\begin{array}{c}\mathbf{5 0} \\
(100)\end{array}$ & $\begin{array}{c}151 \\
(47.1)\end{array}$ & $\begin{array}{c}100 \\
(100)\end{array}$ & $\begin{array}{l}85 \\
(85)\end{array}$ \\
\hline $\begin{array}{l}\text { Riding of spoiled } \\
\text { blood/ dirty from the } \\
\text { body }\end{array}$ & $\begin{array}{c}38 \\
(49.3)\end{array}$ & $\begin{array}{c}32 \\
(38.5)\end{array}$ & $\begin{array}{c}48 \\
(55.1)\end{array}$ & $\begin{array}{c}36 \\
(49.3)\end{array}$ & $\begin{array}{c}86 \\
(52.4)\end{array}$ & $\begin{array}{c}68 \\
(43.5)\end{array}$ & $\begin{array}{c}70 \\
(43.75)\end{array}$ & $\begin{array}{c}127 \\
(79.3)\end{array}$ & $\begin{array}{c}50 \\
(100)\end{array}$ & $\begin{array}{l}30 \\
(60)\end{array}$ & $\begin{array}{c}32 \\
(64)\end{array}$ & $\begin{array}{c}50 \\
(100)\end{array}$ & $\begin{array}{c}197 \\
(61.5)\end{array}$ & $\begin{array}{c}80 \\
(80)\end{array}$ & $\begin{array}{l}82 \\
(82)\end{array}$ \\
\hline $\begin{array}{l}\text { Related to } \\
\text { reproductive system }\end{array}$ & $\begin{array}{c}16 \\
(20.7)\end{array}$ & $\begin{array}{c}42 \\
(50.6)\end{array}$ & $\begin{array}{c}4 \\
(4.5)\end{array}$ & $\begin{array}{c}20 \\
(27.3)\end{array}$ & $\begin{array}{c}20 \\
(12.1)\end{array}$ & $\begin{array}{c}62 \\
(39.7)\end{array}$ & $\begin{array}{c}58 \\
(36.2)\end{array}$ & $\begin{array}{c}24 \\
(15)\end{array}$ & $\begin{array}{c}\mathbf{5 0} \\
(100)\end{array}$ & $\begin{array}{c}31 \\
(62)\end{array}$ & $\begin{array}{c}6 \\
(12)\end{array}$ & $\begin{array}{c}39 \\
(78)\end{array}$ & $\begin{array}{c}82 \\
(25.6)\end{array}$ & $\begin{array}{c}81 \\
(81)\end{array}$ & $\begin{array}{c}45 \\
(45)\end{array}$ \\
\hline Hormonal changes & $\begin{array}{c}12 \\
(15.5)\end{array}$ & $\begin{array}{c}24 \\
(28.9) \\
\end{array}$ & $\begin{array}{c}3 \\
(3.4)\end{array}$ & $\begin{array}{c}21 \\
(28.7)\end{array}$ & $\begin{array}{l}15 \\
(9.1)\end{array}$ & $\begin{array}{c}45 \\
(28.8) \\
\end{array}$ & $\begin{array}{c}36 \\
(22.5)\end{array}$ & $\begin{array}{l}24 \\
(15)\end{array}$ & - & - & - & - & $\begin{array}{c}60 \\
(18.7)\end{array}$ & - & - \\
\hline $\begin{array}{l}\text { Bleeding of } \\
\text { abdominal wounds }\end{array}$ & $\begin{array}{c}12 \\
(15.5)\end{array}$ & $\begin{array}{c}20 \\
(24.0)\end{array}$ & $\begin{array}{c}4 \\
(4.5)\end{array}$ & - & $\begin{array}{c}16 \\
(9.7)\end{array}$ & $\begin{array}{c}20 \\
(12.8)\end{array}$ & $\begin{array}{l}32 \\
(20)\end{array}$ & $\begin{array}{c}4 \\
(2.5)\end{array}$ & - & - & - & - & $\begin{array}{c}36 \\
(11.2)\end{array}$ & - & - \\
\hline $\begin{array}{l}\text { Supematural } \\
\text { phenomena (curse) }\end{array}$ & $\begin{array}{c}2 \\
(2.5)\end{array}$ & $\begin{array}{c}4 \\
(4.8) \\
\end{array}$ & - & $\begin{array}{c}3 \\
(4.1) \\
\end{array}$ & $\begin{array}{c}2 \\
(1.2)\end{array}$ & $\begin{array}{c}7 \\
(4.4)\end{array}$ & $\begin{array}{c}6 \\
(3.75) \\
\end{array}$ & $\begin{array}{c}3 \\
(1.5) \\
\end{array}$ & - & - & - & - & $\begin{array}{c}3 \\
(0.9) \\
\end{array}$ & - & - \\
\hline Don't Know & $\begin{array}{c}5 \\
(6.49)\end{array}$ & $\begin{array}{c}1 \\
(1.20)\end{array}$ & $\begin{array}{c}17 \\
(19.5)\end{array}$ & $\begin{array}{c}8 \\
(10.9)\end{array}$ & $\begin{array}{c}22 \\
(13.4)\end{array}$ & $\begin{array}{c}9 \\
(5.7)\end{array}$ & $\begin{array}{c}6 \\
(3.7)\end{array}$ & $\begin{array}{c}25 \\
(15.6)\end{array}$ & - & - & $\begin{array}{c}12 \\
(24)\end{array}$ & - & $\begin{array}{c}31 \\
(9.6)\end{array}$ & - & $\begin{array}{c}12 \\
(12)\end{array}$ \\
\hline
\end{tabular}


**Multiple Responses

All (100\%) mothers and majority (85\%) of grandmothers held incorrect perception, regarding the causes of menstruation. They reported that, aging is the main cause behind menstruation; they reported that, after crossing a certain age, menstruation occur in girls. This perception was also shared by $47.11 \%$ of the adolescent girls. Another, $61.5 \%$ of adolescent girls, $80 \%$ of mothers and $82 \%$ of grandmothers assumed that, menstruation occurs in females, in order to get rid of spoiled or dirty blood from the body, and it helps to detoxify our blood. Similar results were reported by Gupta \& Gupta (2001); and Nagar \& Aimol (2010). 25.6\% adolescent girls, $81 \%$ of mother and $45 \%$ of grandmothers felt that, it is related to the reproductive system, as it means that, girls become mature and capable to reproduce, once they started menstruating. They also reported that, those females, who don't have menstruation beyond a certain age, may have some problems associated with their reproductive system. Only $18.7 \%$ of the girls knew that, it occurs due to hormonal change, but none of the mothers and grandmothers reported this. $11.2 \%$ of the sample girls (20\% of Purig tribe and $2.5 \%$ from Balti tribe) felt that, it is a bleeding from the abdominal area, 0.9\% of girls believed, it as a curse of God and remaining $9.6 \%$ of girls and $12 \%$ of grandmothers had no idea about why this happens. The results indicate that, the females of Kargil did not have adequate knowledge about the causative factors, leading to occurrence of menstruation and the majority of them, continue to be uncertain about why it occurs.

\section{KNOWLEDGE ABOUT AVERAGE AGE AT MENARCHE}

Table 2: Knowledge about Average Age at Menarche

\begin{tabular}{|c|c|c|c|c|c|c|c|c|c|c|c|c|c|c|c|}
\hline \multirow[b]{2}{*}{$\begin{array}{l}\text { Age in } \\
\text { Year }\end{array}$} & \multicolumn{2}{|c|}{ Purig Girls } & \multicolumn{2}{|c|}{ Balti Girls } & \multirow{2}{*}{$\begin{array}{c}\begin{array}{c}\text { Early } \\
\text { Ado. }\end{array} \\
14-16 \\
\text { Years } \\
N=164\end{array}$} & \multirow{2}{*}{$\begin{array}{c}\begin{array}{c}\text { Late } \\
\text { Ado }\end{array} \\
16-18 \\
\text { Years } \\
\mathrm{N}=156\end{array}$} & \multicolumn{2}{|c|}{ Girls } & \multicolumn{2}{|c|}{ Mothers } & \multicolumn{2}{|c|}{ Grandmothers } & \multicolumn{3}{|c|}{ Females } \\
\hline & $\begin{array}{c}14-16 \\
N=77 \\
(\%)\end{array}$ & $\begin{array}{c}16-18 \\
N=83 \\
(\%)\end{array}$ & $\begin{array}{c}14-16 \\
N=87 \\
(\%)\end{array}$ & $\begin{array}{c}16-18 \\
N=73 \\
(\%)\end{array}$ & & & $\begin{array}{l}\text { Purig } \\
\mathrm{N}=160\end{array}$ & $\begin{array}{c}\text { Balti } \\
\mathrm{N}=160\end{array}$ & \begin{tabular}{|c|} 
Puri \\
$\mathrm{g}$ \\
$\mathrm{N}=5$ \\
0
\end{tabular} & $\begin{array}{l}\text { Balti } \\
\mathrm{N}=\mathbf{5 0}\end{array}$ & $\begin{array}{l}\text { Purig } \\
\mathrm{N}=\mathbf{5 0}\end{array}$ & $\begin{array}{r}\text { Balti } \\
\mathrm{N}=\mathbf{5 0}\end{array}$ & $\begin{array}{c}\text { Girls } \\
\mathrm{N}=320\end{array}$ & $\begin{array}{c}\text { Mothers } \\
\mathrm{N}=100\end{array}$ & $\begin{array}{l}\text { Grand } \\
\text { Mothers } \\
\mathrm{N}=100\end{array}$ \\
\hline $\begin{array}{l}9-11 \\
\text { years }\end{array}$ & - & - & $\begin{array}{c}3 \\
(3.4) \\
\end{array}$ & $\begin{array}{c}1 \\
(1.3)\end{array}$ & $\begin{array}{c}3 \\
(1.8) \\
\end{array}$ & $\begin{array}{c}1 \\
(0.6)\end{array}$ & - & $\begin{array}{c}4 \\
(2.5) \\
\end{array}$ & - & - & - & - & $\begin{array}{c}4 \\
(1.25) \\
\end{array}$ & - & - \\
\hline $\begin{array}{l}12 \\
\text { years }\end{array}$ & $\begin{array}{c}10 \\
(12.9)\end{array}$ & $\begin{array}{c}19 \\
(22.8) \\
\end{array}$ & $\begin{array}{c}4 \\
(4.5) \\
\end{array}$ & $\begin{array}{c}2 \\
(2.7) \\
\end{array}$ & $\begin{array}{c}14 \\
(8.5) \\
\end{array}$ & $\begin{array}{c}21 \\
(13.4)\end{array}$ & $\begin{array}{c}29 \\
(18.1)\end{array}$ & $\begin{array}{c}6 \\
(3.75) \\
\end{array}$ & - & - & - & - & $\begin{array}{c}35 \\
(10.9) \\
\end{array}$ & - & - \\
\hline $\begin{array}{l}13 \\
\text { years }\end{array}$ & $\begin{array}{c}6 \\
(7.7) \\
\end{array}$ & $\begin{array}{c}25 \\
(30.1)\end{array}$ & $\begin{array}{c}34 \\
(39) \\
\end{array}$ & $\begin{array}{c}32 \\
(43.8)\end{array}$ & $\begin{array}{c}40 \\
(24.3)\end{array}$ & $\begin{array}{c}57 \\
(36.5)\end{array}$ & $\begin{array}{c}31 \\
(19.3)\end{array}$ & $\begin{array}{c}66 \\
(41.2) \\
\end{array}$ & - & - & - & - & $\begin{array}{c}97 \\
(30.3)\end{array}$ & - & - \\
\hline $\begin{array}{l}14 \\
\text { years }\end{array}$ & $\begin{array}{c}2 \\
(2.5) \\
\end{array}$ & $\begin{array}{c}8 \\
(9.6) \\
\end{array}$ & $\begin{array}{c}27 \\
(31)\end{array}$ & $\begin{array}{c}5 \\
(6.8) \\
\end{array}$ & $\begin{array}{c}29 \\
(17.6) \\
\end{array}$ & $\begin{array}{c}13 \\
(8.3) \\
\end{array}$ & $\begin{array}{c}10 \\
(6.25)\end{array}$ & $\begin{array}{c}32 \\
(20) \\
\end{array}$ & - & - & - & - & $\begin{array}{c}42 \\
(13.1)\end{array}$ & - & - \\
\hline $\begin{array}{l}\text { Betwee } \\
\text { n } 9-16 \\
\text { years }\end{array}$ & $\begin{array}{c}17 \\
(22.0)\end{array}$ & $\begin{array}{c}22 \\
(26.5)\end{array}$ & $\begin{array}{c}1 \\
(1.1)\end{array}$ & $\begin{array}{c}15 \\
(20.5)\end{array}$ & $\begin{array}{c}18 \\
(10.9)\end{array}$ & $\begin{array}{c}37 \\
(23.7)\end{array}$ & $\begin{array}{c}39 \\
(24.3)\end{array}$ & $\begin{array}{l}16 \\
(10)\end{array}$ & $\begin{array}{c}50 \\
(100 \\
(\end{array}$ & $\begin{array}{c}50 \\
(100)\end{array}$ & $\begin{array}{c}50 \\
(100)\end{array}$ & $\begin{array}{c}50 \\
(100)\end{array}$ & $\begin{array}{c}55 \\
(17.1)\end{array}$ & $\begin{array}{c}100 \\
(100)\end{array}$ & $\begin{array}{l}100 \\
(100)\end{array}$ \\
\hline \multirow[t]{2}{*}{$\begin{array}{l}\text { Uncerta } \\
\text { in }\end{array}$} & $\begin{array}{c}42 \\
(54.5)\end{array}$ & $\begin{array}{c}9 \\
(10.8)\end{array}$ & $\begin{array}{c}18 \\
(20.6)\end{array}$ & $\begin{array}{c}18 \\
(24.6)\end{array}$ & $\begin{array}{c}60 \\
(36.5)\end{array}$ & $\begin{array}{c}27 \\
(17.3)\end{array}$ & $\begin{array}{c}51 \\
(31.8)\end{array}$ & $\begin{array}{c}36 \\
(22.5)\end{array}$ & - & - & - & - & $\begin{array}{c}87 \\
(27.1)\end{array}$ & - & - \\
\hline & \multicolumn{6}{|c|}{$\begin{array}{l}\text { Chi-sq between early and late adolescent girls } 30.3^{* *}, p=0.00 \text {, } \\
\mathrm{df}=5\end{array}$} & \multicolumn{2}{|c|}{$\begin{array}{l}\text { Chi-sq between } \\
\text { Purig and Balti girls } \\
49.5^{* *}, \mathrm{p}=0.00 \text {, } \\
\mathrm{df} 5\end{array}$} & \multicolumn{2}{|l|}{ - - } & \multicolumn{2}{|l|}{-} & \multicolumn{3}{|c|}{$\begin{array}{l}\text { Chi-sq across generations } 32 * 2, \mathrm{~F} \\
=0.00, \mathrm{df} 10\end{array}$} \\
\hline
\end{tabular}

All sample mothers (100\%) and grandmothers (100\%), but only 17\% (24.3\% of Purig tribe and $10 \%$ from Balti tribe) of the adolescent girls were aware that, menarche can occur anywhere between 9-16 years. According to these respondents as menarche signifies reproductive maturity, it can occur anytime in the age group of 9-16 years. On the other hand, the girl respondents gave specific ages, at which menarche occurs. 30\% of girls (19.3\% of Purig tribe and $41.2 \%$ from Balti tribe) reported that, 13 years is an average age for menarche, 13\% of girls reported 14 years and remaining $10 \%$ reported it to be 12 years. However, $27 \%$ of girls (31.8\% of Purig tribe and $22.5 \%$ from Balti tribe) were still not sure about normal age of menarche. Expectedly, the older generation females were more aware about the average age at menarche, than the younger generation and the statistical analysis also shows highly significant difference, across the three generations. When analysed between early adolescent girls and late adolescent girls, highly significant differences between 
them were also seen, as more of late adolescent girls were more aware about this, than early adolescent girls.

Tribe wise analysis shows that, comparatively Purig tribe girls were more aware than Balti tribe girls. More Purig tribe girls $(24.3 \%)$ reported correctly that, it is normal if menarche occurs in girls anywhere between 9-16 years, whereas the majority of Balti tribe girls reported that, 13 years is an ideal age for menarche. However, no significant differences were noted between mothers; and grandmothers of two tribes, as both the groups forwarded similar responses.

\section{ORGAN OF ORIGIN OF MENSTRUAL FLOW}

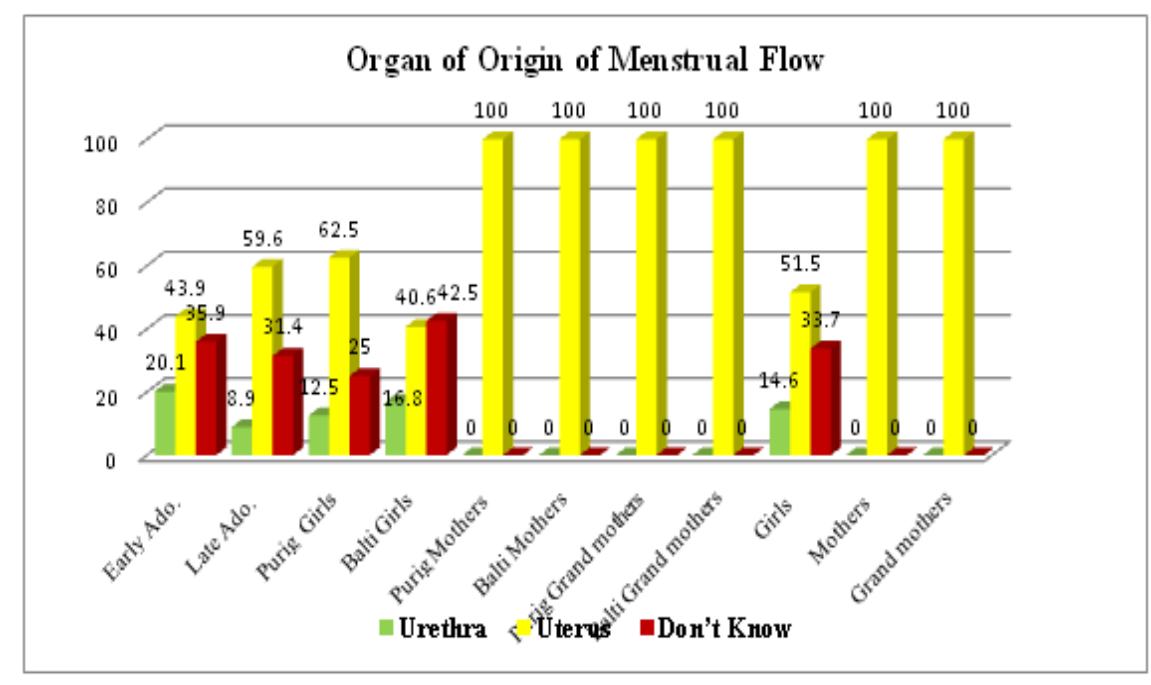

Figure: 1

The respondent's knowledge, regarding the place from where menstrual blood flows out was correctly reported as uterus, by most (51\%) adolescent girls, all (100\%) mothers and (100\%) grandmothers of both the tribes. However, it was striking to note that, $33.7 \%$ of girls were still not sure about the place from where menstrual blood comes. These girls were not able to differentiate between urethra and vaginal opening, and remaining $14.6 \%$ of the girls answered wrongly, by reporting urethra as a source of menstrual blood. The results again reveal a low level of knowledge about female physiology, among adolescent girls of Kargil district. Such issues are least discussed, by the elder females of the family and also, the girls felt uncomfortable and shy, to ask about such topic. Hesitation suppressed their urge to know about such matter probably, because of cultural silence against verbalizing anything, related to sex or sex organ. In a study conducted by Singh (2006), among adolescent girls of Varanasi district (UP), only $43.5 \%$ of the girls correctly responded uterus, as the organ from where the menstrual blood comes. Similarly, in the study conducted by Adhikari (2007), among the rural adolescent girls of Nepal, $25.3 \%$ of the girls reported uterus as the organ from where the bleeding comes, whereas $32 \%$, $26.7 \%$ and $16 \%$ said the Fallopian tube, vagina and urinary bladder, respectively. Similarly, the findings highlight the lack of awareness among adolescent girls, about menstrual flow and the reproductive physiology.

Statistical analysis also shows a highly significant difference across the three generations, as older females were more aware; and between early adolescent girls and late adolescent girl, late adolescents were aware than early adolescent girls.

Tribe wise, no differences were noted for mothers' and grandmothers' knowledge, related to origin of menstrual blood. However, a highly significant difference was found between Purig girls and Balti girls, with more girls of Purig tribe tracing that, menstrual blood comes to the uterus (62.5\%), than the awareness of Balti tribe girls $(40.6 \%)$. 


\section{AVERAGE DURATION OF MENSTRUAL FLOW}

Table 3: Average Duration of Menstrual Flow

\begin{tabular}{|c|c|c|c|c|c|c|c|c|c|c|c|c|c|c|c|}
\hline \multirow{2}{*}{$\begin{array}{l}\text { Average } \\
\text { Length in } \\
\text { Days }\end{array}$} & \multicolumn{2}{|c|}{ Purig Girls } & \multicolumn{2}{|c|}{ Balti Girls } & \multirow{2}{*}{$\begin{array}{c}\begin{array}{c}\text { Early } \\
\text { Ado. }\end{array} \\
14-16 \\
\text { Years } \\
\mathrm{N}=164\end{array}$} & \multirow{2}{*}{$\begin{array}{c}\begin{array}{c}\text { Late } \\
\text { Ado }\end{array} \\
16-18 \\
\text { Years } \\
\mathrm{N}=156\end{array}$} & \multicolumn{2}{|c|}{ Girls } & \multicolumn{2}{|c|}{ Mothers } & \multicolumn{2}{|c|}{ Grandmothers } & \multicolumn{3}{|c|}{ Females } \\
\hline & $\begin{array}{c}14-16 \\
N=77 \\
(\%) \\
\end{array}$ & $\begin{array}{c}16-18 \\
N=83 \\
(\%) \\
\end{array}$ & $\begin{array}{c}14-16 \\
N=87 \\
(\%) \\
\end{array}$ & $\begin{array}{c}16-18 \\
N=73 \\
(\%) \\
\end{array}$ & & & $\begin{array}{l}\text { Purig } \\
\mathrm{N}=160\end{array}$ & $\begin{array}{c}\text { Balti } \\
\mathrm{N}=160\end{array}$ & $\begin{array}{l}\text { Purig } \\
\mathrm{N}=50\end{array}$ & $\begin{array}{l}\text { Balti } \\
\mathrm{N}=50\end{array}$ & $\begin{array}{l}\text { Purig } \\
\mathrm{N}=50\end{array}$ & $\begin{array}{l}\text { Balti } \\
\mathrm{N}=50\end{array}$ & $\begin{array}{c}\text { Girls } \\
\mathrm{N}=320\end{array}$ & $\begin{array}{l}\text { Mothers } \\
N=100\end{array}$ & $\begin{array}{c}\text { Grand } \\
\text { Mothers } \\
\mathrm{N}=100\end{array}$ \\
\hline 3-5 days & $\begin{array}{c}30 \\
(38.9)\end{array}$ & $\begin{array}{c}6 \\
(7.2)\end{array}$ & $\begin{array}{c}14 \\
(16) \\
\end{array}$ & $\begin{array}{c}9 \\
(12.3)\end{array}$ & $\begin{array}{c}44 \\
(26.8) \\
\end{array}$ & $\begin{array}{c}15 \\
(9.6)\end{array}$ & $\begin{array}{c}36 \\
(22.5)\end{array}$ & $\begin{array}{c}23 \\
(14.3)\end{array}$ & - & - & - & - & $\begin{array}{c}59 \\
(18.4)\end{array}$ & - & - \\
\hline 5-7 days & $\begin{array}{c}37 \\
(48.0) \\
\end{array}$ & $\begin{array}{c}67 \\
(80.7) \\
\end{array}$ & $\begin{array}{c}49 \\
(56.3)\end{array}$ & $\begin{array}{c}45 \\
(61.6) \\
\end{array}$ & $\begin{array}{c}86 \\
(52.4)\end{array}$ & $\begin{array}{c}112 \\
(71 \%)\end{array}$ & $\begin{array}{l}104 \\
(65)\end{array}$ & $\begin{array}{c}94 \\
(58.7)\end{array}$ & - & - & - & - & $\begin{array}{c}198 \\
(61.8)\end{array}$ & - & - \\
\hline $7-9$ days & $\begin{array}{c}2 \\
(2.5)\end{array}$ & $\begin{array}{c}4 \\
(4.81)\end{array}$ & $\begin{array}{c}17 \\
(19.5)\end{array}$ & $\begin{array}{c}15 \\
(20.5)\end{array}$ & $\begin{array}{c}19 \\
(11.5)\end{array}$ & $\begin{array}{c}19 \\
(12.1)\end{array}$ & $\begin{array}{c}6 \\
(3.75)\end{array}$ & $\begin{array}{c}32 \\
(20)\end{array}$ & - & - & - & - & $\begin{array}{c}38 \\
(11.8)\end{array}$ & - & - \\
\hline 9-11 days & $\begin{array}{c}2 \\
(2.5)\end{array}$ & $\begin{array}{c}4 \\
(4.81) \\
\end{array}$ & $\begin{array}{c}7 \\
(8)\end{array}$ & $\begin{array}{c}4 \\
(5.4)\end{array}$ & $\begin{array}{c}9 \\
(5.4)\end{array}$ & $\begin{array}{c}8 \\
(5.1)\end{array}$ & $\begin{array}{c}6 \\
(3.7)\end{array}$ & $\begin{array}{c}11 \\
(6.8)\end{array}$ & - & - & - & - & $\begin{array}{c}17 \\
(5.3)\end{array}$ & - & - \\
\hline $\begin{array}{l}\text { Any of } \\
\text { the above }\end{array}$ & - & - & - & - & - & - & - & - & $\begin{array}{c}50 \\
(100)\end{array}$ & $\begin{array}{c}50 \\
(100)\end{array}$ & $\begin{array}{c}50 \\
(100)\end{array}$ & $\begin{array}{c}50 \\
(100)\end{array}$ & - & $\begin{array}{c}100 \\
(100)\end{array}$ & $\begin{array}{c}100 \\
(100)\end{array}$ \\
\hline $\begin{array}{l}\text { I am not } \\
\text { sure }\end{array}$ & $\begin{array}{c}6 \\
(7.7) \\
\end{array}$ & $\begin{array}{c}2 \\
(2.4)\end{array}$ & - & - & $\begin{array}{c}6 \\
(3.6) \\
\end{array}$ & $\begin{array}{c}2 \\
(1.2)\end{array}$ & $\begin{array}{c}8 \\
(5) \\
\end{array}$ & - & - & - & - & - & $\begin{array}{c}8 \\
(2.5)\end{array}$ & - & - \\
\hline & \multicolumn{6}{|c|}{$\begin{array}{l}\text { Chi-sq between early and late adolescent girls } 19.5, p=0.000^{* *} \text {, } \\
\mathrm{df}=4\end{array}$} & \multicolumn{2}{|c|}{$\begin{array}{l}\text { Chi-sq between Purig } \\
\text { and Balti girls } 30.6 \text {, } \\
\mathrm{p}=0.000^{* 8} \text {, } \\
\mathrm{df}=4\end{array}$} & \multicolumn{2}{|l|}{ - } & \multicolumn{2}{|l|}{ - } & \multicolumn{2}{|c|}{$\begin{array}{l}\text { Chi-sq } \\
503, \mathrm{p}=0, \mathrm{df}=10\end{array}$} & generations \\
\hline
\end{tabular}

All sample mothers $(100 \%)$ and grandmothers (100\%) precisely reported that, the average duration of menstrual flow can be from 3 days to 11 days. These respondents further reported that, there is actually nothing to fear, if a female had a menstrual flow of minimum 3 days and maximum of 11 days, but if the female had more than 11 days of period, then, it is not a sign of normal flow and there is need to worry and medical treatment is required. On the other hand, the young generation adolescent girls had different views and ideas, about the normal length of menstrual flow. A large number (61.8\%) of girls reported that, 5-7 days is a normal length of menstrual flow, as majority of them experienced it for the same duration. However, 18.4\% reported 3-5 days, followed by11. 8\% mentioning 7-9 days and 5.3\% saying 9-11 days is the average length of menstrual flow. The results reveal that, the older generation women (mothers and grandmothers) from both the tribes had a better understanding, about the average duration of menstrual flow, but contrastingly the young generation (adolescent girls) were ignorant about this. Statistically, significant differences were seen across the three generations and among early adolescent girls and late adolescent girl, in this aspect as well. Tribe wise; significant differences were also seen among the girls.

\section{REMEDIAL MEASURES SUGGESTED FOR DYSMENORRHOEA}

Table 4: Remedial Measures Suggested for Dysmenorrhoea

\begin{tabular}{|c|c|c|c|c|c|c|c|c|c|c|c|c|c|c|c|}
\hline \multirow{2}{*}{$\begin{array}{l}\text { Remedial } \\
\text { Measures }\end{array}$} & \multicolumn{2}{|c|}{ Purig Girls } & \multicolumn{2}{|c|}{ Balti Girls } & \multirow{2}{*}{$\begin{array}{c}\text { Early } \\
\text { Ado. } \\
14-16 \\
\text { Years } \\
\text { N= } 164\end{array}$} & \multirow{2}{*}{$\begin{array}{c}\text { Late } \\
\text { Ado } \\
16-18 \\
\text { Years } \\
N=156\end{array}$} & \multicolumn{2}{|c|}{ Girls } & \multicolumn{2}{|c|}{ Mothers } & \multicolumn{2}{|c|}{ Grandmothers } & \multicolumn{3}{|c|}{ Females } \\
\hline & $\begin{array}{c}14-16 \\
N=77 \\
(\%)\end{array}$ & $\begin{array}{c}16-18 \\
N=83 \\
(\%)\end{array}$ & $\begin{array}{c}14-16 \\
N=87 \\
(\%)\end{array}$ & $\begin{array}{c}16-18 \\
N=73 \\
(\%)\end{array}$ & & & $\begin{array}{l}\text { Purig } \\
N=16\end{array}$ & $\begin{array}{l}\text { Balti } \\
\mathrm{N}=16\end{array}$ & $\begin{array}{l}\text { Purig } \\
N=50\end{array}$ & $\begin{array}{l}\text { Balti } \\
\mathrm{N}=50\end{array}$ & $\begin{array}{l}\text { Purig } \\
\mathrm{N}=50\end{array}$ & $\begin{array}{l}\text { Balti } \\
N=50\end{array}$ & $\begin{array}{c}\text { Girls } \\
\mathrm{N}=320\end{array}$ & $\begin{array}{c}\text { Mothers } \\
N=100\end{array}$ & $\begin{array}{c}\text { Grand } \\
\text { Mothers } \\
\mathrm{N}=100\end{array}$ \\
\hline $\begin{array}{l}\text { Dietary } \\
\text { changes }\end{array}$ & $\begin{array}{c}18 \\
(23.3)\end{array}$ & $\begin{array}{c}36 \\
(43.3)\end{array}$ & $\begin{array}{c}30 \\
(41)\end{array}$ & $\begin{array}{c}17 \\
(23.2)\end{array}$ & $\begin{array}{c}48 \\
(29.2)\end{array}$ & $\begin{array}{c}53 \\
(33.9)\end{array}$ & $\begin{array}{c}54 \\
(33.7)\end{array}$ & $\begin{array}{c}47 \\
(29.3)\end{array}$ & $\begin{array}{c}23 \\
(46)\end{array}$ & $\begin{array}{c}18 \\
(36)\end{array}$ & $\begin{array}{c}30 \\
(60)\end{array}$ & $\begin{array}{c}25 \\
(50)\end{array}$ & $\begin{array}{c}101 \\
(31.5)\end{array}$ & $\begin{array}{c}41 \\
(41)\end{array}$ & $\begin{array}{c}55 \\
(55)\end{array}$ \\
\hline Exercise & $\begin{array}{c}2 \\
(2.5) \\
\end{array}$ & $\begin{array}{c}4 \\
(4.8) \\
\end{array}$ & $\begin{array}{c}12 \\
(13.7)\end{array}$ & $\begin{array}{c}6 \\
(8.2) \\
\end{array}$ & $\begin{array}{c}14 \\
(8.5) \\
\end{array}$ & $\begin{array}{c}10 \\
(6.4)\end{array}$ & $\begin{array}{c}6 \\
(3.75) \\
\end{array}$ & $\begin{array}{c}18 \\
\text { (11.2) }\end{array}$ & - & $\begin{array}{c}24 \\
(48) \\
\end{array}$ & - & $\begin{array}{c}13 \\
(26) \\
\end{array}$ & $\begin{array}{c}24 \\
(7.5) \\
\end{array}$ & $\begin{array}{c}24 \\
(24) \\
\end{array}$ & $\begin{array}{c}13 \\
(13)\end{array}$ \\
\hline Medicines & $\begin{array}{c}12 \\
(15.5)\end{array}$ & $\begin{array}{c}46 \\
(54.1)\end{array}$ & $\begin{array}{c}18 \\
(20.6)\end{array}$ & $\begin{array}{c}3 \\
(3.6)\end{array}$ & $\begin{array}{c}30 \\
(18.2)\end{array}$ & $\begin{array}{c}49 \\
(31.4)\end{array}$ & $\begin{array}{c}58 \\
(36.2)\end{array}$ & $\begin{array}{c}21 \\
(13.1)\end{array}$ & $\begin{array}{c}9 \\
(18)\end{array}$ & $\begin{array}{c}12 \\
(24)\end{array}$ & & & $\begin{array}{c}79 \\
(24.6)\end{array}$ & $\begin{array}{c}21 \\
\text { (21) }\end{array}$ & - \\
\hline $\begin{array}{l}\text { Home base } \\
\text { treatments }\end{array}$ & $\begin{array}{c}31 \\
(40.2)\end{array}$ & $\begin{array}{c}43 \\
((51.8)\end{array}$ & $\begin{array}{c}11 \\
(12.6)\end{array}$ & $\begin{array}{c}40 \\
(54.7)\end{array}$ & $\begin{array}{c}42 \\
(25.6)\end{array}$ & $\begin{array}{c}83 \\
(53.2)\end{array}$ & $\begin{array}{c}74 \\
(46.2)\end{array}$ & $\begin{array}{c}51 \\
(31.8)\end{array}$ & $\begin{array}{c}23 \\
(46)\end{array}$ & - & $\begin{array}{c}30 \\
(60)\end{array}$ & $\begin{array}{c}6 \\
(12)\end{array}$ & $\begin{array}{l}125 \\
(39)\end{array}$ & $\begin{array}{l}23 \\
\text { (23) }\end{array}$ & $\begin{array}{l}36 \\
(36)\end{array}$ \\
\hline Not sure & - & - & $\begin{array}{c}17 \\
(19.5)\end{array}$ & $\begin{array}{c}10 \\
(13.6)\end{array}$ & $\begin{array}{c}17 \\
(10.3)\end{array}$ & $\begin{array}{c}10 \\
(6.4)\end{array}$ & - & $\begin{array}{c}27 \\
(16.8)\end{array}$ & - & - & - & - & $\begin{array}{c}27 \\
(8.4) \\
\end{array}$ & - & - \\
\hline Nothing & $\begin{array}{c}32 \\
(41.5)\end{array}$ & $\begin{array}{c}8 \\
(9.6)\end{array}$ & $\begin{array}{c}9 \\
(10.3)\end{array}$ & $\begin{array}{c}7 \\
(9.5)\end{array}$ & $\begin{array}{c}41 \\
(25)\end{array}$ & $\begin{array}{c}15 \\
(9.6)\end{array}$ & $\begin{array}{c}40 \\
(25)\end{array}$ & $\begin{array}{l}16 \\
(10\end{array}$ & $\begin{array}{c}33 \\
(66)\end{array}$ & $\begin{array}{c}20 \\
(40)\end{array}$ & $\begin{array}{c}20 \\
(40)\end{array}$ & - & $\begin{array}{c}56 \\
(17.5)\end{array}$ & $\begin{array}{c}53 \\
(53)\end{array}$ & $\begin{array}{c}20 \\
(20)\end{array}$ \\
\hline
\end{tabular}

**Multiple responses 
With respect to the respondents' knowledge level, regarding the remedial measures for Dysmenorrhoea across the three generations, majority of adolescent girls, believed in efficiency of home base treatments (39\%) like resting and placing of hot water bags on lower abdomen, $23 \%$ mothers and 36\% grandmothers also shared the same views. However, the majority of mothers (53\%) did not believe in making use of any remedy, for menstrual pain. They reported that, they endured Dysmenorrhoea in the hope that, it is a matter of only a few days. The same was felt by $17.5 \%$ girls and $20 \%$ grandmothers. A majority of grandmothers $(55 \%)$ reported that, change in diet was often recommended. Women were told to reduce the intake of cold and sour foods like curd and apricot, in order to minimize Dysmenorrhoea and were advised to increase the intake of hot foods like local butter tea, with barley flour and leafy vegetables. They further reported the practice of taking a half cup of melted butter, if the pain is severe, as butter is considered as hot food and it helps to flush out the menstrual blood, from the uterus. Rest, $7.5 \%$ of girls, $27 \%$ of mothers and $13 \%$ of grandmothers said that, simple exercise like walking is also recommended during periods, as walking again helps to shed out the blood more quickly from the uterus. Only $24 \%$ of girls and $21 \%$ of mothers said that, medicines are the best remedy for Dysmenorrhoea.

\section{DYSMENORRHOEA AMONG GIRLS AS ABNORMAL}

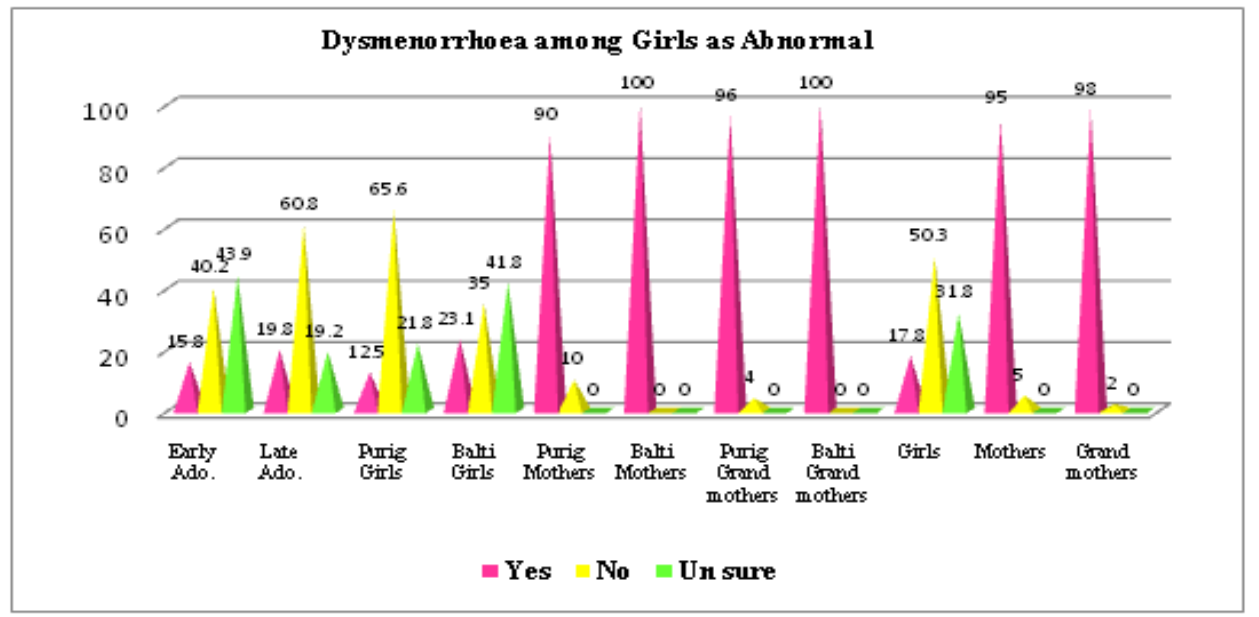

Figure: 2

Qualitative findings revealed that, among the three generations almost all the mother (95\%) and grandmother (98\%) participants commonly believed that, Dysmenorrhoea in girls is not normal. They further stated that, those females who do not have menstruation after a certain age may have some problems, associated with the reproductive system. On the other hand, the majority (50\%) of girls correctly reported that, it is untrue that Dysmenorrhoea in girls is not normal, $31.8 \%$ of girls were not sure about this and only $17.8 \%$ agreed that, it is true. Statistical analysis showed that, there is a highly significant difference $(\mathrm{p}=0.000)$ across the three generations, and also between early and late adolescent girls, on this concept.

On the basis of tribes, no significant difference was seen between mothers and grandmothers of Purig and Balti tribe, as both the groups viewed Dysmenorrhoea in girls is abnormal, whereas (65.6\%) Purig girls and (35\%) Balti girls did not think of approving it. 


\section{AWARENESS ABOUT AVERAGE AGE AT MENOPAUSE}

Table 5: Awareness about Average Age at Menopause

\begin{tabular}{|c|c|c|c|c|c|c|c|c|c|c|c|c|c|c|c|}
\hline \multirow[b]{2}{*}{$\begin{array}{c}\text { Age } \\
\text { (Years) }\end{array}$} & \multicolumn{2}{|c|}{ Purig Girls } & \multicolumn{2}{|c|}{ Balti Girls } & \multirow{2}{*}{$\begin{array}{c}\text { Early } \\
\text { Ado. } \\
14-16 \\
\text { Years } \\
N=164\end{array}$} & \multirow{2}{*}{$\begin{array}{c}\begin{array}{c}\text { Late } \\
\text { Ado }\end{array} \\
16-18 \\
\text { Years } \\
\text { N=156 }\end{array}$} & \multicolumn{2}{|c|}{ Girls } & \multicolumn{2}{|c|}{ Mothers } & \multicolumn{2}{|c|}{ Grandmothers } & \multicolumn{3}{|c|}{ Females } \\
\hline & $\begin{array}{c}14-16 \\
N=70 \\
(\%)\end{array}$ & $\begin{array}{c}16-18 \\
N=78 \\
(\%)\end{array}$ & $\begin{array}{c}14-16 \\
N=50 \\
(\%)\end{array}$ & $\begin{array}{c}16-18 \\
N=70 \\
(\%)\end{array}$ & & & $\begin{array}{l}\text { Purig } \\
N=16\end{array}$ & $\begin{array}{c}\text { Balti } \\
N=16\end{array}$ & $\begin{array}{l}\text { Purig } \\
\mathrm{N}=50\end{array}$ & $\begin{array}{l}\text { Balti } \\
\mathbf{N}=\mathbf{5 0}\end{array}$ & $\begin{array}{l}\text { Purig } \\
\mathbf{N}=\mathbf{5 0}\end{array}$ & $\begin{array}{l}\text { Balti } \\
\mathbf{N}=\mathbf{5 0}\end{array}$ & $\begin{array}{c}\text { Girls } \\
N=320\end{array}$ & $\begin{array}{c}\text { Mothers } \\
\mathrm{N}=100\end{array}$ & $\begin{array}{c}\text { Grand } \\
\text { Mothers } \\
\mathrm{N}=100\end{array}$ \\
\hline $40+-45$ & $\begin{array}{c}14 \\
(18.1)\end{array}$ & $\begin{array}{c}18 \\
(21.6)\end{array}$ & $\begin{array}{c}2 \\
(2.2)\end{array}$ & $\begin{array}{c}21 \\
(28.7)\end{array}$ & $\begin{array}{c}16 \\
(9.7)\end{array}$ & $\begin{array}{c}39 \\
(25)\end{array}$ & $\begin{array}{c}32 \\
(20)\end{array}$ & $\begin{array}{c}23 \\
(14.3)\end{array}$ & - & - & - & - & $\begin{array}{c}\mathbf{5 5} \\
(17.1)\end{array}$ & - & - \\
\hline $45+-50$ & $\begin{array}{c}34 \\
(44.1)\end{array}$ & $\begin{array}{c}50 \\
(60.2)\end{array}$ & $\begin{array}{c}12 \\
(13.7)\end{array}$ & $\begin{array}{c}31 \\
(42.4)\end{array}$ & $\begin{array}{c}46 \\
(28)\end{array}$ & $\begin{array}{c}81 \\
(51.9)\end{array}$ & $\begin{array}{c}84 \\
(52.5)\end{array}$ & $\begin{array}{c}43 \\
(26.8)\end{array}$ & $\begin{array}{c}30 \\
(60)\end{array}$ & $\begin{array}{c}38 \\
(76)\end{array}$ & $\begin{array}{c}16 \\
(32)\end{array}$ & $\begin{array}{c}44 \\
(88)\end{array}$ & $\begin{array}{c}127 \\
(39.6)\end{array}$ & $\begin{array}{c}68 \\
(68)\end{array}$ & $\begin{array}{c}60 \\
(60)\end{array}$ \\
\hline $50+-55$ & $\begin{array}{c}8 \\
(10.3)\end{array}$ & & $\begin{array}{c}10 \\
(11.4)\end{array}$ & $\begin{array}{c}5 \\
(6.8)\end{array}$ & $\begin{array}{c}18 \\
(10.9)\end{array}$ & $\begin{array}{c}5 \\
(3.2)\end{array}$ & $\begin{array}{c}8 \\
(5)\end{array}$ & $\begin{array}{c}15 \\
(9.3)\end{array}$ & $\begin{array}{c}15 \\
(30)\end{array}$ & $\begin{array}{c}12 \\
(24)\end{array}$ & $\begin{array}{l}25 \\
(50)\end{array}$ & $\begin{array}{c}6 \\
(12)\end{array}$ & $\begin{array}{c}23 \\
(7.1)\end{array}$ & $\begin{array}{c}27 \\
(27)\end{array}$ & $\begin{array}{c}31 \\
(31)\end{array}$ \\
\hline $55+-60$ & - & - & $\begin{array}{c}17 \\
(19.5)\end{array}$ & $\begin{array}{c}9 \\
(12.3)\end{array}$ & $\begin{array}{c}17 \\
(10.3)\end{array}$ & $\begin{array}{c}9 \\
(5.7)\end{array}$ & - & $\begin{array}{c}26 \\
(16.2)\end{array}$ & - & - & $\begin{array}{c}6 \\
(12)\end{array}$ & - & $\begin{array}{c}26 \\
(8.12)\end{array}$ & & $\begin{array}{c}6 \\
6\end{array}$ \\
\hline $60+$ & $\begin{array}{c}14 \\
(18.1)\end{array}$ & $\begin{array}{c}10 \\
\text { (12) }\end{array}$ & $\begin{array}{c}9 \\
(10.7)\end{array}$ & $\begin{array}{c}2 \\
(2.7)\end{array}$ & $\begin{array}{c}23 \\
(14)\end{array}$ & $\begin{array}{c}12 \\
(7.6)\end{array}$ & $\begin{array}{l}24 \\
(15)\end{array}$ & $\begin{array}{c}11 \\
(6.8)\end{array}$ & $\begin{array}{c}\mathbf{5} \\
(10)\end{array}$ & - & $\begin{array}{c}3 \\
(6)\end{array}$ & - & $\begin{array}{c}35 \\
(10.9)\end{array}$ & $\begin{array}{c}5 \\
(5)\end{array}$ & $\begin{array}{c}3 \\
(3)\end{array}$ \\
\hline \multirow[t]{2}{*}{$\begin{array}{l}\text { Not } \\
\text { Sure }\end{array}$} & & $4(4.81)$ & - & $2(2.7)$ & - & - & $\begin{array}{c}4 \\
(2.5)\end{array}$ & $\begin{array}{c}13 \\
(8.12)\end{array}$ & - & - & - & - & $\begin{array}{c}23 \\
(7.1)\end{array}$ & - & - \\
\hline & \multicolumn{5}{|c|}{$\begin{array}{l}\text { Chi-sq between ear } \\
p=0.00, d f=4\end{array}$} & $30.2^{* *}$, & \multicolumn{2}{|c|}{$\begin{array}{l}\text { Chi-sq between } \\
\text { Purig and Balti } \\
\text { Grandmothers } \\
51.1, p=0, d f=5\end{array}$} & \multicolumn{2}{|c|}{$\begin{array}{l}\text { Chi-sq between } \\
\text { Purig and Balti } \\
\text { Grandmothers } \\
6.2, p=0.04 \text {, } \\
d f=2\end{array}$} & \multicolumn{2}{|c|}{$\begin{array}{l}\text { Chi-sq between } \\
\text { Purig and Balti } \\
\text { Grandmothers } \\
28.102, \quad p=2.3 \mathrm{e}- \\
7, \mathrm{df}=3\end{array}$} & \multicolumn{3}{|c|}{$\begin{array}{l}\text { Chi-sq across generation } \\
114.1^{* *}, \mathrm{p}=0, \mathrm{df}=10\end{array}$} \\
\hline
\end{tabular}

The majority (68\%) of mothers and (60\%) grandmothers stated that, menopause occurs somewhere between 46-50 years, followed by 51-55 years, by $27 \%$ mothers and $31 \%$ grandmothers. It was also noted that, in their culture they calculated their age in the cycle. Each cycle consist of 12 years, so majority of respondents had a concept that, the menopause occurs or is attained after crossing $4^{\text {th }}$ year cycle of their age, that means $(12 \times 4) 48$ years onwards and before crossing $5^{\text {th }}$ year of their age cycle, $(12 \times 5=60) 60$ years. They also reported that, it is also affected by ones fitness, health, diet and heredity. However, a large number of girls did not know about this 'age cycle', so they had different ideas and views about age at menopause. The majority (39.6\%) of the adolescent girls reported that, it stops between 46-50 years, $17.1 \%$ stated, $40-45$ years of age, $10 \%$ of girls were of the idea that, it stops after crossing 60 years and remaining $7.1 \%$ of girls were undecided. This reveals that, older females had better knowledge about menopause, probably because of their age and experience. Tribe wise Purig girls had better awareness, than Balti girls and statistically significant differences were found between them.

\section{CONCLUSIONS}

Expectedly, the knowledge level of mother and grandmothers regarding menstruation was far better than the young adolescent girls naturally, because they were well experienced with it. On the whole, it can be concluded that, the young adolescent girls of Kargil did not have adequate knowledge about menstruation and female reproductive physiology. The majority of them didn't know exactly, the causative factors leading to occurrence of menstruation and some were still not sure about the place from where menstrual blood comes. Similarly, there were many related aspects, where the girls showed a poor concept and lacked basic knowledge. This is probably because, such issues are least discussed by the elder females, within the family. The mothers and grandmothers didn't communicate about menstruation with their daughters and granddaughters, leading to lack of continuity in their knowledge. Most of the mother and grandmothers of Kargil were not playing a constructive role, in preparing their daughters and granddaughters for menstruation. In fact, they themselves felt uncomfortable and nervous, about discussing menstruation with their daughters and hence, were secretive and uncommunicative. Due to this, misconceptions and wrong perceptions grew within the mind of young adolescent girls. Therefore, it is highly recommended to educate the girls, about the facts of menstruation, physiological implications and significance of menstruation, at an early age. For this, the mothers need to be empowered with necessary skills, to communicate and transfer the information to their adolescent girls, to bring positive change in their Knowledge and also 
develop a healthy relationship between mothers and daughters.

\section{REFERENCES}

1. Adhikari, P., Kadel, B., Dhungel, S., \& Mandal, A. (2007). Knowledge and Practice Regarding Menstrual Hygiene In Rural Adolescent Girls of Nepal. Kathmandu University Medical Journal, 5(3), 382-386.

2. Gupta, J., \& Gupta, H. (2001). Adolescents and Menstruation. The Journal of Family Welfare, 47(1), 1-13. Harlow, D., \& Cambpbell, O. (2004). Epidemiology of Menstrual disorders in developing countries: A Systematic review. British Journal of Obstetrics and Gynaecology, 111, 6-16

3. Kamaljit, K., Arora, B., Singh, G. K., \& Neki, N. S. (2012). Social Beliefs and Practices associated with Menstrual Hygiene among Adolescent Girls of Amritsar, Punjab. Journal of International Medical Sciences Academy, 25(2), 69-70.

4. Nagar, S., \& Aimol, R. (2010). Knowledge of Adolescent Girls Regarding Menstruation in Tribal Areas of Meghalaya. Tribes Tribals, 8(1), 27-30.

5. Singh, A. J. (2006). The place of menstruation in the reproductive lives of women of rural north India. Indian Journal of Community Medicine, 31(1), 10-14.

6. Shokry, E., Allah, A., Elsayed, E., \& Elsabagh, M. (2011). Impact of Health Education Intervention on Knowledge and practice about Menstruation among Female Secondary School Students in Zagazig City. Journal of American Science 7(9), 737-747. 